\section{Concentrações de 25-hidroxivitamina $D$ e níveis pressóricos em idosos hipertensos}

\author{
25-hydroxyvitamin D concentrations and blood \\ pressure levels in hypertensive elderly patients
}

Juliana Padilha Ramos Neves', Alexandre Sérgio Silva², Liana Clébia Soares Lima de Morais², Alcides da Silva Diniz ${ }^{3}$, Maria José de Carvalho Costa², Luiza Sonia Rios Asciutti ${ }^{4}$, Maria da Conceição Rodrigues Gonçalves ${ }^{2}$

\section{RESUMO}

Objetivo: Avaliar fatores relacionados à prevalência da hipovitaminose $D$ e relação com pressão arterial em 91 idosos hipertensos de João Pessoa, PB/BR. Materiais e métodos: Em estudo transversal, níveis de 25-hidroxivitamina $\mathrm{D}$ foram comparados com dados bioquímicos, antropométricos, dietéticos, tipo de pele, exposição solar e pressão arterial. Resultados: A prevalência da inadequação da vitamina (25-hidroxivitamina $D<29 \mathrm{ng} / \mathrm{mL}$ ) foi $33 \%$. A concentração sérica de 25-hidroxivitamina $D$ associou-se inversamente com a pressão arterial sistólica e positivamente com frequência semanal do consumo de peixes. As outras variáveis estudadas não mostraram associação significativa com 25-hidroxivitamina D. Conclusão: A prevalência da inadequação das concentrações de vitamina $D$ foi elevada e relacionada com maior pressão arterial em idosos. Por outro lado, um maior consumo semanal de peixe está ligado a maiores concentrações da 25-hidroxivitamina D. Arq Bras Endocrinol Metab. 2012;56(7):415-22

\section{Descritores}

Idosos; deficiência de vitaminas; vitamina D; hipertensão
${ }^{1}$ Pós-graduação em Ciências da Nutrição, Centro de Ciências da Saúde, Universidade Federal da Paraíba (UFPB), João Pessoa, PB, Brasil ${ }^{2}$ Programa de Pós-graduação em Ciências da Nutrição, Departamento de Nutrição, Centro de Ciências da Saúde, UFPB, João Pessoa, PB, Brasil ${ }^{3}$ Departamento de Nutrição, Centro de Ciências da Saúde, Universidade Federal de Pernambuco (UFPE), Recife, PE, Brasil ${ }^{4}$ Faculdade de Ciências Médicas da Paraíba, Curso de Graduação em Nutrição, João Pessoa, PB, Brasil

Correspondência para: Juliana Padilha Ramos Neves Av. Governador Juvenal Lamartine, 326 , ap. 102, Torre-I, Tirol 59022-020 - Natal, RN, Brasil juliana.prn@ig.com.br

Recebido em 7/Maio/2012 Aceito em 24/Ago/2012 was inversely associated with systolic blood pressure and positively associated with weekly fish consumption. The other variables showed no significant association with 25-hydroxyvitamin D. Conclusion: The prevalence of inadequate vitamin D concentrations was related with higher blood pressure in elderly patients. Moreover, greater fish weekly consumption influenced greater 25-hydroxyvitamin D concentration. Arq Bras Endocrinol Metab. 2012;56(7):415-22

\title{
Keywords
}

Elderly patients; vitamin deficiency; vitamin D; hypertension

\section{INTRODUÇÃO}

\footnotetext{
A alterações fisiológicas inerentes ao envelhecimento Apodem contribuir para o surgimento de complicações clínicas, entre essas, aquelas relacionadas aos baixos níveis de vitamina D. Deficiência de vitamina $\mathrm{D}$ pode ser explicada no idoso pela redução da capacidade de gerar
}

seu precursor na pele, o 7-de-hidrocolesterol que se transforma em vitamina D3 pela ação dos raios ultravioleta $B$, pelo uso diário e necessário do protetor solar, mudança de estilo de vida e redução de atividades físicas ao ar livre (1).

Níveis inadequados da 25-hidroxivitamina D implicam diminuição do cálcio sérico pela redução da absor- 
ção intestinal desse mineral, que, por sua vez, implica hiperestimulação da glândula paratireoide a liberar $\mathrm{PTH}$, a fim de elevar a reabsorção renal e óssea de cálcio (2).

A vitamina $\mathrm{D}$ vem sendo reconhecida como responsável por vários efeitos biológicos, muito além do metabolismo do cálcio e homeostase óssea, apresentando propriedades anti-inflamatórias (3), influenciando na modulação da resistência à insulina, a qual é mediada via receptor de vitamina $\mathrm{D}(4)$, e mostrando-se como um potente supressor da biossíntese de renina que funciona como fator coadjuvante no controle da pressão arterial (5).

De fato, estudos mostram associação inversa entre os níveis séricos de vitamina $\mathrm{D}$ e pressão arterial ou atividade da renina plasmática em normotensos e hipertensos (6). Judd e cols. (7) investigaram o efeito do tratamento da vitamina D sobre a hipertensão arterial em humanos por três semanas e observaram diminuição da pressão arterial sistólica em comparação ao grupo placebo. Essa relação inversa entre vitamina $\mathrm{D}$ e hipertensão arterial foi confirmada nos estudos de seguimento Health Professionals Follow-up Study e Nurse's Health Study (8).

Muitos desses estudos foram conduzidos na Europa, nos Estados Unidos, em países orientais, entre outros, mas não existem ainda na literatura dados relacionando níveis de vitamina $\mathrm{D}$ com hipertensão arterial no Brasil, os quais poderiam elucidar ainda melhor essa associação, considerando a alta incidência solar no Brasil durante todo o ano, pela sua proximidade à linha do Equador, sendo a exposição solar um fator a partir do qual o indivíduo obtém a vitamina D3.

Os estudos de prevalência da hipovitaminose $\mathrm{D}$ em idosos no Brasil envolveram idosos institucionalizados, ambulatoriais e mulheres menopausadas. Encontrou-se, em alguns estudos, prevalência de 71\% nos idosos institucionalizados e 56\% nos ambulatoriais em São Paulo (9), 86\% também em idosos institucionalizados no Rio Grande do Sul (10) e 68\% em menopausadas ambulatoriais em Recife (11). As menores prevalências foram em estudos com idosos ambulatoriais, enquanto as maiores prevalência foram verificadas com idosos institucionalizados. Daí a necessidade de melhor investigar fatores intervenientes como exposição ao sol, cor da pele e ingestão de alimentos fontes de vitamina $\mathrm{D}$, que foram considerados em alguns, mas não em todos esses estudos anteriores. Além disso, essa associação entre hipovitaminose D e hipertensão arterial verificada em outros países não foi estudada no Brasil, sendo essa informação importante ao se considerar que aproximadamente 10 milhões de brasileiros idosos são hiperten$\operatorname{sos}(12)$.

Diante dessas lacunas ainda persistentes, este trabalho foi conduzido para estimar a prevalência de hipovitaminose $\mathrm{D}$ em idosos hipertensos e sua relação com níveis pressóricos, bem como sua associação com parâmetros bioquímicos, antropométricos, dietéticos, tipo de pele e exposição ao sol.

\section{MATERIAIS E MÉTODOS}

Tratou-se de um estudo de corte transversal realizado na cidade de João Pessoa, PB, localizada no nordeste do Brasil. Existem 50 Centros de Referência e Cidadania nesta cidade, que são cadastrados no Programa de Atenção Básica à Pessoa Idosa (PAPI) da Secretaria do Desenvolvimento Social (Sedes) da Prefeitura. Esses centros estão distribuídos em 31 bairros da cidade. Para este estudo, foram escolhidos, aleatoriamente, seis centros de seis bairros distintos, entre aqueles, os que permitiram a execução do estudo. Os critérios de inclusão da pesquisa foram: idade igual ou superior a 60 anos, com diagnóstico prévio de hipertensão, fazendo obrigatoriamente uso de terapia medicamentosa, não fazendo uso de suplementos que contivessem a vitamina $\mathrm{D}$, não relatassem doença renal, ou qualquer outra doença crônica consumptiva, com estado cognitivo preservado, não etilistas nem tabagistas. A coleta de dados foi realizada no período de maio a agosto do ano de 2011.

Para cálculo amostral, foi utilizada como referência uma prevalência de $56 \%$ de hipovitaminose $\mathrm{D}$ em idosos ambulatoriais em São Paulo (9), um erro amostral de $10 \%$ e nível de confiança de $95 \%$. A amostra calculada foi de 78 idosos e, para corrigir eventuais perdas, trabalhamos com 91 idosos.

O protocolo deste estudo foi previamente aprovado (Protocolo CEP/HULW n ${ }^{\circ}$ 684/10) pelo Comitê de Ética em Pesquisa do Hospital Universitário Lauro Wanderley (CEP/HULW) da Universidade Federal da Paraíba, de acordo com a Resolução n 196/96 do CNS/MS, que regulamenta a ética da pesquisa em seres humanos no Brasil. Todos os sujeitos envolvidos na seleção da amostra foram esclarecidos quanto aos propósitos e métodos do estudo e, ao consentir em participar, assinaram o Termo de Consentimento Livre e Esclarecido. Os casos de hipovitaminose D foram encaminhados para atendimento nas Unidades de Saúde do Município. 
Todos os dados da pesquisa foram coletados nos próprios Centros com questionário elaborado e aplicado pelos pesquisadores. Para evitar vieses de memória, os idosos respondiam ao questionário com o auxílio de acompanhantes que com eles residissem. O questionário continha as seguintes informações:

\section{Fototipo da pele e exposição ao sol}

O fototipo da pele foi classificado como I a VI, segundo Toda e cols. (1973) e referido por Astner e Anderson em 2004 (13). Foi interrogado ao idoso sobre a descrição da sua pele, se queimava com facilidade, pouco, raramente ou nunca, e ainda sobre sua sensibilidade ao sol, variando do pouco sensível ao muito sensível. Com essas informações, o fototipo da pele era classificado variando da cor branca (I) à cor negra (VI). Os sujeitos também foram interrogados quanto ao tempo de exposição ao sol, se este era de pelo menos 30 minutos por dia e se permaneciam mais tempo dentro ou fora de casa durante o dia.

\section{Pressão arterial}

A pressão arterial foi aferida com os idosos sentados e duas horas após o acordar. A medida foi feita pelo método oscilométrico, utilizando-se um esfigmomanômetro aneroide, previamente calibrado contra um de coluna de mercúrio, e seguindo-se rigorosamente o protocolo proposto pelas VI Diretriz de Hipertensão Arterial (14).

\section{Estado nutricional}

A avaliação do estado nutricional foi determinada pelo índice de massa corporal (IMC), calculado a partir da razão entre o peso atual $(\mathrm{kg})$ e o quadrado da altura $\left(\mathrm{m}^{2}\right)$, de acordo com a classificação da WHO (15), em que IMC $<18,5 \mathrm{~kg} / \mathrm{m}^{2}$ classifica-se como baixo peso, $18,5-24,9 \mathrm{~kg} / \mathrm{m}^{2}$ como eutróficos, $25-29,9 \mathrm{~kg} / \mathrm{m}^{2}$ como sobrepeso e IMC $\geq 30 \mathrm{~kg} / \mathrm{m}^{2}$ como obesidade.

$\mathrm{O}$ peso foi verificado em balança digital (modelo BAL-20 PM com capacidade para $150 \mathrm{~kg}$ e intervalo de $100 \mathrm{~g}$ ), com o idoso descalço e com roupas leves. A altura de todos os idosos foi estimada por meio da fórmula do joelho determinada por Chunlea e cols. (16), tendo em vista que a altura do idoso é afetada pela perda óssea e pelo arqueamento da coluna.

\section{Consumo de alimentos fontes de vitamina D}

O inquérito alimentar foi realizado por meio do "Questionário Quantitativo de Frequência do Consumo Ali- mentar" (QQFCA), validado a partir de três recordatórios de 24 horas aplicados em diferentes intervalos de tempo, para uma população de mulheres no município de João Pessoa/PB/Brasil, em parceria com a Faculdade de Saúde Pública da Universidade de São Paulo e o Programa de Pós-Graduação em Ciências da Nutrição da Universidade Federal da Paraíba $(17,18)$. A partir de então, foi feita a quantificação da frequência do consumo semanal de ovos, peixes, leite desnatado e leite integral. A classificação do consumo foi: nunca, às vezes (1-3x/ semana) e frequentemente ( $\geq 4 \mathrm{x} /$ semana), sendo que, para o leite, o consumo era também classificado como diário.

\section{Avaliação laboratorial}

Os idosos foram informados da necessidade do jejum de $12 \mathrm{~h}$ para uma coleta de sangue por punção venosa, que foi realizada no próprio local das reuniões de cada centro por um profissional devidamente habilitado. Todas as análises foram feitas em um laboratório especializado para as análises bioquímicas propostas. Foram dosados no soro: 25-hidroxivitamina $\mathrm{D}$, feito em duplicata pelo método da quimioluminescência (Diasorin LIAISON) automatizado, com coeficientes de variabilidade intra $\mathrm{e}$ intermétodos de $4 \%$ e $6 \%$, respectivamente, considerando os valores de referência de $<20 \mathrm{ng} / \mathrm{mL}$ como deficiência e entre 21-29 ng/mL como insuficiência (19); cálcio ionizado, pelo método calculado considerando os valores de referência de 4,0-5,0 ng/mL; paratormônio, pelo método eletroquimioluminescência com o EDTA (T. Roxa), considerando os valores de referência de 15-65 pg/mL; magnésio pelo método colorimétrico - Bechman Coulter - LX 20, considerando os valores de referência entre 9-2,5 mg/dL; creatinina pelo método Cinético Automatizado - Bechman Coulter - LX 20 considerando os valores de referência para homens de $0,6-1,2 \mathrm{mg} / \mathrm{dL}$ e, para mulheres, de 0,5-1,0 mg/ $\mathrm{dL}$; albumina pelo método Verde de Brocromocresol, considerando como valores de referência 3,5 a $5,5 \mathrm{~g} /$ dL; e glicose pelo método Enzimático Colorimétrico Bechman Coulter - LX 20, considerando como valores de referência entre 60-99 mg/dL.

A função renal dos idosos foi avaliada pela estimação do clearance de creatinina, calculado a partir da creatinina sérica (Crs) utilizando a fórmula de Cockocroft $\mathrm{e}$ Gault (20), sendo considerados como alteração da função renal valores de referência $<60 \mathrm{~mL} / \mathrm{min} / 1,73 \mathrm{~m}^{2}$. 
Foram considerados portadores de hiperparatiroidismo secundário aqueles indivíduos que possuíam PTH acima da normalidade na presença de valores de cálcio ionizado normais.

\section{Análise estatística}

Técnicas de estatística descritiva foram utilizadas mediante distribuições absolutas, percentuais, médias e desvio-padrão (DP). A normalidade e a homogeneidade dos dados foram previamente avaliadas por meio do teste de Kolmogorov-Smirnov. A comparação entre médias de duas variáveis contínuas foi realizada por meio do Teste T-Student para dados não pareados. Para influência do tipo de pele e frequência do consumo de alimentos fonte de vitamina $\mathrm{D}$ sobre os níveis séricos da 25-hidroxivitamina $\mathrm{D}$, foi utilizada análise de variância (ANOVA - one way). Valor de $\mathrm{p}<0,05$ foi considerado estatisticamente significativo. Todos os testes foram realizados no programa Graph Pad Prism 4.0.

\section{RESULTADOS}

A amostra foi de 91 idosos com idade média de 69,73 $(7,0)$ anos, sendo 81 (89\%) mulheres e 10 (11\%) homens. Todos eram hipertensos e utilizavam tratamento medicamentoso com um ou mais fármacos.

De acordo com o critério adotado pela última $\mathrm{Di}$ retriz da Endocrine Society em 2011 (19), a prevalência de níveis inadequados (deficientes e insuficientes) da 25-hidroxivitamina D foi de 33\% ( $\mathrm{n}=30)$, estando quatro deles deficientes (25-hidroxivitamina $\mathrm{D}<20$ $\mathrm{ng} / \mathrm{mL}$ ) e 26 com insuficiência (25-hidroxivitamina D entre 21-29 ng/mL). A média da 25-hidroxivitamina $\mathrm{D}$ no grupo com níveis adequados da vitamina foi de $44,8(12,5) \mathrm{ng} / \mathrm{mL}$ e, no grupo com níveis inadequados, foi $24,1(3,5) \mathrm{ng} / \mathrm{mL}$. Os valores médios de todos os parâmetros bioquímicos segundo status da 25-hidroxivitamina D estão apresentados na tabela 1 . Entre todos os parâmetros bioquímicos analisados, $\mathrm{o}$ cálcio e a creatinina mostraram-se significativamente maiores no grupo dos idosos com níveis inadequados da 25-hidroxivitamina $\mathrm{D}$, entretanto, convém mencionar que não ocorreram valores fora do ponto de corte de cada parâmetro em toda a amostra. Analisando descritivamente, 13 idosos (14,3\%) apresentaram hiperparatiroidismo secundário, com níveis de $\mathrm{PTH}$ elevado e cálcio ionizado normais, dos quais $7(7,7 \%)$
Tabela 1. Parâmetros bioquímicos segundo status da 25-hidroxivitamina $\mathrm{D}$ em idosos hipertensos pertencentes a seis Centros de Referência e Cidadania da Cidade de João Pessoa, PB, Brasil, 2011, expressos em média (DP)

\begin{tabular}{|c|c|c|c|}
\hline & $\begin{array}{c}\text { Idosos } \\
\text { Níveis } \\
\text { adequados da } \\
\text { 25-hidroxivitamina } \\
\text { D* }\end{array}$ & $\begin{array}{c}\text { Idosos } \\
\text { Níveis } \\
\text { inadequados da } \\
\text { 25-hidroxivitamina } \\
\text { D** }\end{array}$ & $\mathbf{p}^{1}$ \\
\hline PTH (pg/mL) & $47,08(14,0)$ & $52,99(18,8)$ & 0,090 \\
\hline Ca-i (ng/dL) & $4,54(0,2)$ & $4,40(0,2)$ & 0,004 \\
\hline Creatinina (ng/dL) & $0,83(0,2)$ & $0,76(0,1)$ & 0,027 \\
\hline Albumina (g/dL) & $4,46(0,4)$ & $4,43(0,4)$ & 0,940 \\
\hline Glicose (ng/dL) & $101,2(43,4)$ & $95,33(23,3)$ & 0,488 \\
\hline Magnésio (mg/dL) & $1,95(0,2)$ & $1,99(0,1)$ & 0,186 \\
\hline $\begin{array}{l}\text { Clearance } \\
\left(\mathrm{mL} / \mathrm{min} / 1,73 \mathrm{~m}^{2}\right)\end{array}$ & $70,12(19,5)$ & $75,43(24,8)$ & 0,06 \\
\hline
\end{tabular}

1 Valores de $\mathrm{p}$ referentes à comparação entre o grupo de idosos com níveis adequados e 0 grupo de níveis inadequados da 25 -hidroxivitamina D, segundo teste T-Student para dados não pareados.

* 25 -hidroxivitamina D $>29 \mathrm{ng} / \mathrm{mL}$; * 25 -hidroxivitamina D $\leq 29 \mathrm{ng} / \mathrm{mL}$.

também apresentaram níveis inadequados da 25-hidroxivitamina D.

Houve maior prevalência $(40,7 \%, \mathrm{n}=37)$ do fototipo da pele IV referida como morena moderada, seguida do III $(24,2 \%, \mathrm{n}=22)$ como morena clara, do II $(16,5 \%, \mathrm{n}=15)$ branca sensível, $\mathrm{V}(13,2 \%, \mathrm{n}=12)$ morena escura, e VI $(5,5 \%, \mathrm{n}=5)$ negra. Não foi encontrada qualquer associação estatística entre o tipo de pele e a condição adequada ou inadequada da 25 -hidroxivitamina D (ANOVA, $\mathrm{p}=0,96$ ).

A maioria dos idosos (71\%) referia se expor ao sol por pelo menos 30 minutos ao dia e $28 \%$ referiram que passam mais tempo fora de casa durante o dia. Não houve relação significativa do tempo de exposição ao sol $(\mathrm{p}=0,7 \mathrm{l})$ e da permanência ou não em casa durante o dia $(\mathrm{p}=0,39)$ com os níveis de 25 -hidroxivitamina $\mathrm{D}$ nos grupos de idosos com níveis adequados e inadequados da vitamina.

Quanto às variáveis nutricionais, a média de peso foi de $66,3(11,7) \mathrm{kg}$ e da altura estimada foi de 1,62 $(6,6) \mathrm{m}$. No diagnóstico nutricional pelo índice de massa corporal, segundo WHO (1998), foram encontrados os seguintes resultados: $4,4 \%(n=4)$ estavam com baixo peso, $45,1 \%(\mathrm{n}=41)$ eutróficos, $35,2 \%(\mathrm{n}=32)$ com sobrepeso e $15,4 \%(n=14)$ com obesidade. Não houve diferenças significantes dos níveis da 25-hidroxivitamina $\mathrm{D}$ entre os grupos adequados e inadequados da vitamina $(\mathrm{p}=0,91)$.

$\mathrm{O}$ consumo de alimentos fontes de vitamina $\mathrm{D}$ mostrou-se interveniente nos níveis séricos da 25-hidroxi- 
Tabela 2. Concentrações séricas da 25-hidroxivitamina $D$ em função da frequência do consumo semanal ${ }^{\star}$ de ovos, peixes, leite integral e desnatado de idosos hipertensos pertencentes a seis Centros de Referência e Cidadania da Cidade de João Pessoa, PB, Brasil, 2011, expressos em média (DP)

\begin{tabular}{llcc}
\hline Consumo & Frequência & $\begin{array}{c}\text { Média } \\
(\mathbf{n g} / \mathbf{m L})\end{array}$ & $\mathbf{p}^{\mathbf{1}}$ \\
\hline Ovos & Nunca $(n=21)$ & $40,5(16,4)$ & 0,476 \\
& Às vezes $(n=48)$ & $36,3(12,2)$ & \\
Peixes & Frequentemente $(n=22)$ & $39,2(16,5)$ & \\
& Nunca $(n=17)$ & $32,4(12,4)^{\mathrm{a}}$ & 0,006 \\
& Às vezes $(\mathrm{n}=57)$ & $36,9(12,4)$ & \\
Leite integral & Frequentemente $(n=17)$ & $47,1(18,2)^{\mathrm{a}}$ & \\
& Nunca $(n=13)$ & $45,2(16,5)$ & 0,053 \\
& Às vezes $(n=5)$ & $41,6(15,8)$ & \\
& Frequentemente $(n=7)$ & $30,3(5,9)$ & \\
& Diariamente $(25)$ & $34,6(12,1)$ & \\
Leite desnatado & Nunca $(n=13)$ & $45,2(16,5)$ & 0,110 \\
& Às vezes $(n=7)$ & $29,2(10,4)$ & \\
& Frequentemente $(n=2)$ & $35,1(2,5)$ & \\
& Diariamente $(19)$ & $43,8(15,3)$ & \\
\hline
\end{tabular}

* Frequência semanal para ovos e peixes: às vezes (1-3x/semana) e frequentemente $(>4 \mathrm{x})$ semana). E para os leites: às vezes (1-3x/semana), frequentemente (4-6x/semana) e diariamente.

1 Valores de p referentes à análise de variância dos níveis de concentração da 25-hidroxivitamina D nas frequências de consumo semanais de ovos, peixes, leite integral e desnatado, segundo ANOVA- one way.

${ }^{a} p<0,05$ segundo pós-teste de Bonferroni.

vitamina D. Na tabela 2, encontram-se os resultados da análise de variância do consumo de ovos, peixes, leite integral e desnatado nos idosos com os níveis da 25-hidroxivitamina D. Um maior consumo de peixe foi positivamente associado com maiores níveis séricos de vitamina $\mathrm{D}(\mathrm{p}=0,006)$. Por outro lado, o consumo de ovo, leite integral e desnatado, alimentos também fonte desse nutriente, não se mostrou influenciar nos níveis de vitamina $\mathrm{D}$ dos idosos deste estudo.

A média de pressão arterial foi de $138,7(22,9)$ $\mathrm{mmHg}$ e $82,9(12,2) \mathrm{mmHg}$ para os valores sistólico e diastólico, respectivamente. Quando a amostra foi separada pelos grupos adequados e inadequados da vitamina $\mathrm{D}$ com os valores pressóricos, a média da pressão arterial sistólica foi significativamente maior $(\mathrm{p}$ $=0,03)$ nos com níveis inadequados da vitamina do que nos adequados, com médias $146(5,3) \mathrm{mmHg} \mathrm{e}$ $135,1(2,4) \mathrm{mmHg}$, respectivamente. Por outro lado, essa mesma relação não ocorreu para pressão diastólica $(\mathrm{p}=0,26)$, com médias $85(2,9) \mathrm{mmHg}$ para os inadequados e $81,97(1,2) \mathrm{mmHg}$ para os adequados da vitamina. A figura 1 mostra a distribuição dos valores pressóricos sistólicos e diastólicos nos grupos.
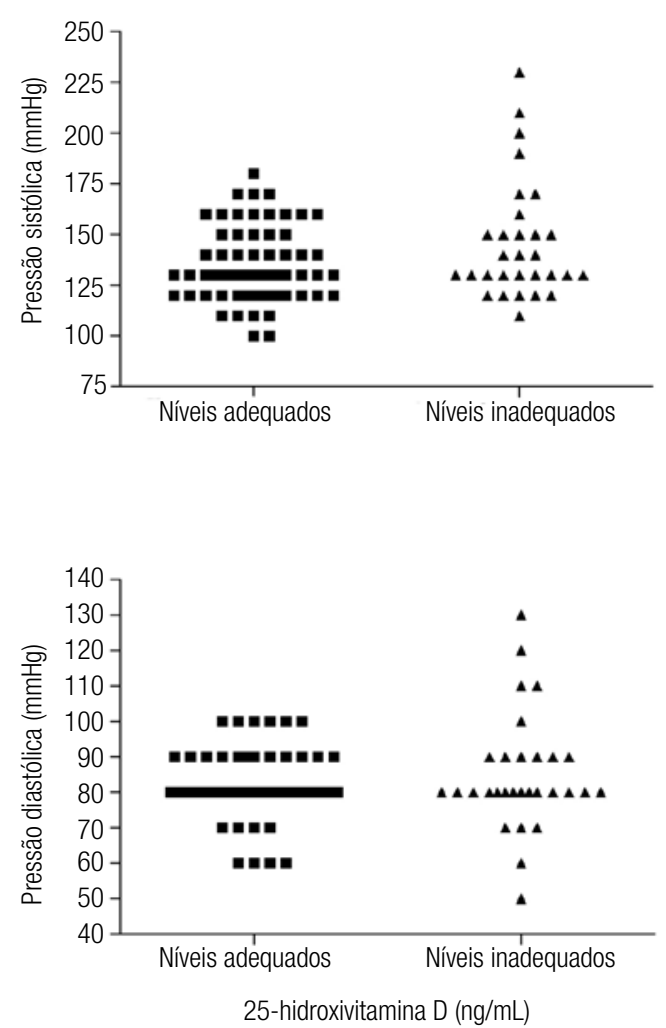

Figura 1. Relação da pressão arterial sistólica e diastólica segundo status da 25-hidroxivitamina D em idosos de seis Centros de Referência e Cidadania da Cidade de João Pessoa, PB, Brasil, 2011.

\section{DISCUSSÃO}

O diagnóstico da prevalência da hipovitaminose $\mathrm{D}$ do presente estudo foi a partir dos pontos de corte adotados pela última Diretriz da Endocrine Society em 2011 (19), em que os sujeitos foram classificados com níveis inadequados da 25-hidroxivitamina $\mathrm{D}$ quando esta se mostrou $<29 \mathrm{ng} / \mathrm{mL}$. No entanto, para comparar com a literatura, é necessário mencionar que não existe uma uniformidade dos pontos de corte. A IOM (21) alerta que, por essa variedade de métodos de medições de pontos de corte, um indivíduo pode ser considerado deficiente ou suficiente da vitamina $\mathrm{D}$, dependendo do laboratório onde é feita a análise. A Endocrine Society (20) manteve o mesmo ponto de corte da IOM de 2010 (21) para determinar deficiência de vitamina D, enquanto Hollis em 2008 (22) classificou um nível mínimo ideal da 25-hidroxivitamina D em $32 \mathrm{ng} / \mathrm{mL}$, ainda assim existem outros pontos de corte adotados nos estudos disponíveis.

Dados da 25-hidroxivitamina D de pós-menopausadas em 25 países de cinco continentes foram analisados e concluíram que $4 \%$ delas eram deficientes da vitami- 
na $\mathrm{D}$ (25-hidroxivitamina $\mathrm{D}<10 \mathrm{ng} / \mathrm{mL})$ e $24 \%$ eram insuficientes (entre 10-20 ng/mL) (23). Outro estudo de grande importância sobre hipovitaminose $\mathrm{D}$ em mulheres com osteoporose com idades entre 41 e 96 anos foi realizado em 18 países incluindo o Brasil (24), onde foram considerados níveis ótimos da 25-hidroxivitamina D entre 20-32 ng/mL. A prevalência de hipovitaminose $\mathrm{D}$ foi de $63,9 \%$, sendo os piores resultados na Turquia e Líbano. No Brasil ( $\mathrm{n}=151)$, a prevalência foi de $42,4 \%$. Nos dois estudos citados, o ponto de corte para níveis adequados da 25-hidroxivitamina $\mathrm{D}$ foi $>20 \mathrm{ng} / \mathrm{mL}$, menor do que o adotado no presente estudo e, mesmo assim, as prevalências foram maiores do que a encontrada. Estudos mais recentes apontam prevalência entre $40 \%$ e $45 \%$ de insuficiência ou deficiência de vitamina D na população Alemã e de 30\% (25-hidroxivitamina D < $30 \mathrm{ng} / \mathrm{mL}$ ) em Chicago, nos Estados Unidos $(25,26)$.

Tomados esses dados em conjunto, pode-se observar que a prevalência de hipovitaminose $\mathrm{D}$ é encontrada em idosos de diferentes continentes independentemente de sua condição $(23)$.

A alta incidência dos raios ultravioleta no Brasil pelos 12 meses do ano é suficientemente elevada para assegurar a produção de vitamina $\mathrm{D}$ na pele humana (27). Neste país, ainda são muito escassos os estudos para diagnóstico da prevalência de hipovitaminose $\mathrm{D}$ em idosos. Porém, mesmo com a alta incidência dos raios ultravioleta, é surpreendente que se encontre prevalência entre $71 \%$ e $86 \%$ de inadequação da 25-hidroxivitamina D em idosos institucionalizados de São Paulo e Porto Alegre, respectivamente $(9,10)$ e entre $49 \%$ e $68 \%$ em mulheres menopausadas, não necessariamente idosas, do Rio de Janeiro e Recife, respectivamente $(28,11)$. Uma das possíveis explicações para a maior prevalência nos idosos institucionalizados é que esses sujeitos podem ser bem menos expostos à luz solar quando comparados às mulheres menopausadas, que teoricamente poderiam ter mais exposição ao sol.

Convém salientar que esses estudos citados foram conduzidos com metodologias diferentes, inclusive com variedades de pontos de corte, de modo que essas prevalências não podem ser seguramente comparadas com nossos resultados observados. Utilizando o mesmo ponto de corte deste estudo e com uma prevalência bem similar, foi encontrada em $42,4 \%$ dos usuários de um ambulatório de endocrinologia de Belo Horizonte, porém os sujeitos tinham idade entre 14 e 91 $\operatorname{anos}(29)$.
Além de idosos terem menor exposição ao sol por apresentarem uma mudança de estilo de vida e redução de atividades físicas ao ar livre (1), uma pele mais velha tem capacidade significativamente menor de sintetizar a vitamina $\mathrm{D}$ pela luz solar do que a pele de pessoas mais jovens (30). Sendo assim, o aumento da produção de vitamina $\mathrm{D}$ pela luz solar não é, portanto, uma opção realista para a maioria dos pacientes geriátricos, ainda que residentes em países ensolarados.

Independentemente do processo do envelhecimento, a melanina funciona como um protetor solar natural, de modo que, quanto mais escura for a pele, maior será sua necessidade de exposição solar para sintetizar a mesma quantidade de vitamina D (31). Porém, neste estudo não houve relação significativa entre os tipos de pele e níveis da 25 -hidroxivitamina $\mathrm{D}$, assim como não houve relação significativa entre tempo de exposição ao sol nem se o idoso permanecia mais tempo ou não em casa. Scalco e cols. (10) também estudaram essas mesmas variáveis em relação aos níveis da 25-hidroxivitamina $\mathrm{D}$ e também não encontraram resultados significativos, porém, a população estudada foi de idosos institucionalizados, nos quais se espera uma menor exposição ao sol. Portanto, nossos dados indicam que somente a radiação solar não é suficiente para determinar os níveis de vitamina D no organismo dos idosos.

Uma relação comumente encontrada com a alta prevalência de hipovitaminose $\mathrm{D}$ é a presença de hiperparatiroidismo secundário, visto que no soro a concentração de PTH é inversamente relacionada à 25-hidroxivitamina D (9). A despeito dessas considerações, o estudo não mostrou relação significativa entre PTH e 25-hidroxivitamina D. O hiperparatiroidismo secundário ocorreu em $14,3 \%$ da amostra e essa condição concomitante com níveis inadequados da vitamina $\mathrm{D}$ aconteceu em $7,7 \%$ da amostra $(\mathrm{n}=7)$, resultados bem diferentes dos achados por Saraiva e cols. (9), em que se encontrou prevalência de $61,7 \%$ nos pacientes do grupo institucionalizado e de $54 \%$ nos pacientes do grupo ambulatorial. Huisman e cols. (32) afirmam que os idosos necessitam de concentrações mais elevadas de 25OHD para normalizarem suas concentrações plasmáticas de PTH.

Recentemente, foi testada a relação entre IMC e concentrações de 25 -hidroxivitamina $\mathrm{D}$ em iranianas de 20 a 64 anos de idade (33), e, igualmente ao presente estudo, não foi verificada relação estatisticamente significativa. Entretanto, Mai e cols., em 2012 (34), obser- 
varam associação significativa entre concentrações $<20$ $\mathrm{ng} / \mathrm{mL}$ com o surgimento de obesidade em adultos.

Com relação à alimentação, existem dados dos Estados Unidos indicando que apenas $2 \%$ da população acima de 70 anos satisfaz suas necessidades diárias de vitamina $\mathrm{D}$ apenas com alimentos, mesmo considerando que o leite americano é enriquecido com vitamina D (35). No Brasil não há essa política de fortificação, limitando ainda mais o consumo de alimentos fonte dessa vitamina. Dessa forma, no presente estudo foi investigada a relação da frequência do consumo semanal de ovos, peixes, leite integral e desnatado com as concentrações da 25-hidroxivitamina D. Essa relação foi estatisticamente significativa para o consumo semanal de peixes $(\mathrm{p}=0,006)$ corroborando com um estudo realizado em mulheres no Japão, país onde também não há política de fortificação, com relação significativa entre a frequência do consumo semanal de ovos e peixes em relação às concentrações da 25-hidroxivitamina D (36). Outro estudo recente nos Estados Unidos (26) identificou que o baixo ou não consumo de peixe estava relacionado com a deficiência da vitamina D. Sabendo que o leite brasileiro não é fortificado, este estudo confirmou que, mesmo o consumo mais frequente do leite integral ou desnatado, não houve relação significativa com os níveis da 25-hidroxivitamina D.

A hipovitaminose $\mathrm{D}$ pode influenciar na pressão arterial por ser um potente supressor do sistema endócrino da biossíntese de renina, que regula o sistema renina-angiotensina (5). Estudos têm demonstrado uma relação inversa entre hipovitaminose $\mathrm{D}$ e níveis pressóricos sistólicos e diastólicos. Entretanto, neste estudo essa relação foi observada apenas com a pressão arterial sistólica. Isso reforça os achados de Judd e cols. ao identificarem que o tratamento da vitamina D sobre a hipertensão arterial em humanos por três semanas acarretou uma diminuição de $9 \%$ da pressão arterial sistólica (7). Relação inversa significativa entre 25-hidroxivitamina $\mathrm{D}$ e pressão arterial sistólica e diastólica também foi confirmada recentemente em homens, mas não em mulheres em Xangai e em uma população indiana $(37,38)$, corroborando com os dados do Health Professionals Follow-up Study (HPFS) e do Nurse's Health Study, nos quais foi possível concluir que os níveis 25-hidroxivitamina D são inversamente associados ao risco de desenvolver hipertensão (8). Todos esses estudos prévios mencionam os mesmos mecanismos pelos quais a vitamina $\mathrm{D}$ reduz a pressão arterial, mas não faz distinção entre a pressão arterial sistólica e diastólica. Para entendimento dos resultados aqui relatados da relação significativa dos níveis inadequados da 25-hidroxivitamina D com apenas a pressão arterial sistólica, fazem-se necessários experimentos futuros.

Mesmo diante dos resultados dos estudos que vêm mostrando essa relação inversa entre vitamina D e pressão arterial, autores ainda alertam que resultados de estudos randomizados de suplementação de vitamina D (com colecalciferol ou ergocalciferol) para baixar a pressão arterial são inconsistentes, possivelmente em decorrência da variabilidade na população do estudo, tamanho da amostra, dose e duração (39).

Embora este estudo tenha demonstrado uma relação inversa significativa entre níveis inadequados da 25-hidroxivitamina D e níveis de pressão arterial sistólica, chamamos a atenção para realização de mais pesquisas para investigar possíveis associações potenciais nas diferenciais sexuais, visto que nossa amostra foi na sua maioria mulheres.

Estudos futuros também poderiam esclarecer melhor outros alimentos fontes de vitamina $\mathrm{D}$ levando em consideração o hábito alimentar brasileiro, assim como as espécies de peixes e o impacto destes nas concentrações séricas da 25-hidroxivitamina $\mathrm{D}$, visto que se observou neste estudo uma relação positiva entre um maior consumo semanal de peixe e níveis da 25 -hidroxivitamina D.

Conclui-se que a inadequação dos níveis de vitamina D mostrou-se elevada na população geriátrica e hipertensa estudada e acredita-se que, pelos resultados aqui mostrados, o consumo de peixe deve ser incentivado. Além disso, faz-se necessário discutir a implementação de políticas de fortificação de alimentos com vitamina D. Essas são as melhores estratégias comparativamente à adoção de suplementação da vitamina, pela inconsistência de indicações de doses relatadas em diversos estudos, incluindo os com suplementação da vitamina D para efeitos benéficos em hipertensos. A hipertensão é um problema de saúde pública no nosso país e, sabendo dos achados positivos da vitamina $\mathrm{D}$ sobre os níveis pressóricos, esta pode ser vista como um coadjuvante no tratamento de hipertensos.

Agradecimentos: à Secretaria de Desenvolvimento Social (Sedes) de João Pessoa, por consentir a realização deste estudo nos Centros de Referência e Cidadania nos quais funcionam os encontros dos idosos cadastrados.

Declaração: os autores declaram não haver conflitos de interesse científico neste estudo. 


\section{REFERÊNCIAS}

1. Lanske B, Razzaque MS. Vitamin D and aging: old concepts and new insights. J Nutr Biochem. 2007;18(12):771-7.

2. Cozzolino SMF. Biodisponibilidade de nutrientes. 3.ed. São Paulo: Manole; 2009.

3. Lai JK, Lucas RM, Clements MS, Harrison SL, Banks E. Assessing vitamin D status: pitfalls for the unwary. Mol Nutr Food Res. 2010;54(8):1062-71.

4. Sugden JA, Davies JI, Witham MD, Morris AD, Struthers AD. Vitamin $D$ improves endothelial function in patients with Type 2 diabetes mellitus and low vitamin D levels. Diabet Med. 2008;25(3):320-5.

5. LiYC, Qiao G, Uskokovic M, Xiang W, Zheng W, Kong J. Vitamin D: a negative endocrine regulator of the renin-angiotensin system and blood pressure. J Steroid Boichem Mol Biol. 2004;89-90(1-5):387-92.

6. Schuch NJ, Garcia VC, Martini LA. Vitamina D e doenças endocrinometabólicas. Arq Bras Endocrinol Metab. 2009;53(5):625-33.

7. Judd SE, Raiser SN, Kumari M,Tangpricha V. 1,25-Dihydroxyvitamin $D(3)$ reduces systolic blood pressure in hypertensive adults: a pilot feasibility study. J Steroid Biochem Mol Biol. 2010;121(1-2):445-7.

8. Forman JP, Giovannucci E, Holmes MD, Bischoff-Ferrari HA, Tworoger SS, WillettWC, et al. Plasma 25-hydroxivitamin D levels and risk incident hypertension. Hypertension. 2007;49(5):1063-9.

9. Saraiva GL, Cendoroglo MS, Ramos LR, Araújo LM, Vieira JG, Maeda SS, et al. Prevalence of vitamin D deficiency, insufficiency and secondary hyperparathyroidism in the elderly inpatients and living in the community of the city of São Paulo, Brazil. Arq Bras Endocrinol Metab. 2007;51(3):437-42.

10. Scalco R, Premaor MO, Fröehlich PE, Furlanetto TW. High prevalence of hypovitaminosis $\mathrm{D}$ and secondary hyperparathyroidism in elders living in nonprofit homes in South Brazil. Endocrine. 2008;33(1):95-100.

11. Bandeira F, Griz L, Freese E, Lima DC, Thé AC, Diniz ET, et al. Vitamin $D$ deficiency and its relationship with bone mineral density among postmenopausal women living in the tropics. Arq Bras Endocrinol Metab. 2010;54(2):227-32.

12. IBGE/Instituto Brasileiro de Geografia e Estatística. Síntese de Indicadores Sociais. 2010. Uma Análise das Condições de Vida da População Brasileira. Disponível em: http://www.ibge.gov.br/ home/estatistica/populacao/condicaodevida/indicadoresminimos/sinteseindicsociais2010/SIS_2010.pdf.

13. Astner S, Anderson RR. Skin phototypes 2003. J Invest Dermatol. 2004;122(2):xxx-xxxi.

14. SBC/SBH/SBN. Sociedade Brasileira de Cardiologia. Sociedade Brasileira de Hipertensão, Sociedade Brasileira de Fisiologia. VI Diretrizes Brasileiras de Hipertensão Arterial. Arq Bras Cardiol. 2010;95(1 supl.1):1-51.

15. World Health Organization. Obesity: preventing and managing the global epidemic. World Health OrganTech Rep Ser. 1998;894:i-xii, 1-253.

16. Chunlea WC, Roche AF, Steinating ML. Estimating stature from knee height for persons 60 to 90 years of age. J Am Geriat Soc. 1985;33(2):116-20.

17. Lima FEL, Latorre MRDO, Costa MJC, Fisiberg RM. Diet and cancer in northeastern Brazil: evaluation ofeating habits and food group consuption in relation to breast cancer. Cad Saúde Pública (Fiocruz). 2008;24(4):820-8.

18. Lima FEL, Slater B, Latorre MRDO, Fisiberg RM. Validade de um questionário quantitativo de frequência alimentar desenvolvido para população feminina no nordeste do Brasil. Rev Bras Epidemiol. 2007;10(4):483-90.

19. Holick MF, Binkley NC, Bischoff-Ferrari HA, Gordon CM, Hanley DA, Heaney RP, et al. Evaluation, treatment, and prevention of vitamin $D$ deficiency: an Endocrine Society clinical practice guideline. J Clin Endocrinol Metab. 2011;96(7):1911-30.
20. Cockcroft DW, Gault MH. Prediction of creatinine clearance from serum creatinine. Nephron. 1976;16:31-41.

21. Institute of Medicine. Dietary Reference Intakes for Calcium and Vitamin D. Washington, DC:The National Academies Press; 2010.

22. Hollis BW. Assessment of vitamin D status and definition of a normal circulating range of 25-hydroxyvitamin D. Curr Opin Endocrinol Diabetes Obes. 2008;15(6):489-94.

23. Lips $P$, Duong $T$, Oleksik $A$, Black $D$, Cummings $S$, Cox $D$, et al. A global study of vitamin $D$ status and parathyroid function in postmenopausal women with osteoporosis: baseline data from the Multiple Outcomes of Raloxifen Evaluation clinical trial. J Clin Endocrinol Metab. 2001;86(3):1212-21.

24. Lips P, Hosking D, Lippuner K, Norquist JM, Wehren L, Maalouf G, et al. The prevalence of vitamin $D$ inadequacy amongst women with osteoporosis: an international epidemiological investigation. J Intern Med. 2006;260(3):245-54.

25. Zittermann A. The estimated benefits of vitamin D for Germany. Mol Nutr Food Res. 2010;54(8):1164-71.

26. Orelind E, Feinglass J, Moran M, Zei CP, Baker DW. Correlates of vitamin $D$ insufficiency in an affluent adult population. South Med J. 2012;105(2):78-81d.

27. Paula de PCM, Ceballos JC. Solar ultraviolet radiation measurements in one of the most populous cities of the world: aspects related to skin cancer cases and vitamin $D$ availability. Photochem Photobiol. 2010;86(2):438-44.

28. Russo, LAT, Gregório LH, Lacativa PG, Marinheiro LP. Concentration of 25-hydroxyvitamin $D$ in postmenopausal women with low bone mineral density. Arq Bras Endocrinol Metab. 2009;53(9):1079-87.

29. Silva BCC, Camargos BM, Fujii JB, Dias EP, Soares MMS. Prevalence of vitamin $D$ deficiency and its correlation with PTH, biochemical bone turnover markers and bone mineral density, among patients from ambulatories. Arq Bras Endocrinol Metab. 2008;52(3):482-8.

30. Trémezaygues L, Reichrath J. Zur Bedeutung des vitamina Stoffwechsels in der Haut humanen. Hautarzt. 2010;61:478-86.

31. Holick MF. Sunlight and vitamin D for bone health and prevention of autoimmune disease, cancers, and cardiovascular disease. Am J Clin Nutr. 2004;80(6):1678S-88S.

32. Huisman AM, White KP, Algra A, Harth M, Vieth R, Jacobs JW, et al. Vitamin D levels in women with systemic lupus erythematosus and fibromyalgia. J Rheumatol. 2001;28(11):2535-9.

33. Baradaran A, Behradmanesh S, Nasri H. Association of body mass index and serum vitamin $D$ level in healthy Iranian adolescents. Endokrynol Pol. 2012;63(1):29-33.

34. Mai XM, Chen Y, Camargo CA Jr, Langhammer A. Cross-sectional and prospective cohort study of serum 25-hydroxyvitamin D level and obesity in adults: the HUNT study. Am J Epidemiol. 2012;175(10):1029-36.

35. Serviço Brasileiro de Respostas Técnicas. Dossiê Técnico Fortificação de Alimentos. Rede de Tecnologia do Rio de Janeiro; 2007.

36. Nakamura K, Nashimoto M, Hori Y, Yamamoto M. Serum 25-hidroxyvitamin $D$ concentration and relate dietary factors in peri- and postmenopausal Japonese women. Am J Clin Nutr. 2000;71:1161-5.

37. Dorjgochoo T, Ou Shu X, Xiang YB, Yang G, Cai Q, Li H, et al. Circulating 25-hydroxyvitamin $\mathrm{D}$ levels in relation to blood pressure parameters and hypertension in the Shanghai Women's and Men's Health Studies. Br J Nutr. 2012;27:1-10.

38. Kota SK, Kota SK, Jammula S, Meher LK, Panda S, Tripathy PR, et al. Renin-angiotensin system activity in vitamin $D$ deficient, obese individuals with hypertension: An urban Indian study. Indian J Endocrinol Metab. 2011;5 Suppl 4:S395-401.

39. Vaidya A, Forman JP. Vitamin D and vascular disease: the current and future status of vitamin $D$ therapy in hypertension and kidney disease. Curr Hypertens Rep. 2012;4(2):111-9. 